\title{
Feasibility of contrast-enhanced coronary artery magnetic resonance angiography using compressed sensing
}

\author{
Kuniaki Hirai ${ }^{1}$, Teruhito Kido ${ }^{1}$, Tomoyuki Kido ${ }^{* *}$ (D, Ryo Ogawa², Yuki Tanabe ${ }^{1}$, Masashi Nakamura', \\ Naoto Kawaguchi ${ }^{1}$, Akira Kurata ${ }^{1}$, Kouki Watanabe ${ }^{3}$, Osamu Yamaguchi ${ }^{4}$, Michaela Schmidt ${ }^{5}$, \\ Christoph Forman ${ }^{5}$ and Teruhito Mochizuki ${ }^{1}$
}

\begin{abstract}
Background: Coronary magnetic resonance angiography (CMRA) is a promising technique for assessing the coronary arteries. However, a disadvantage of CMRA is the comparatively long acquisition time. Compressed sensing (CS) can considerably reduce the scan time. The aim of this study was to verify the feasibility of CS CMRA scanning during the waiting time between contrast injection and late gadolinium enhancement (LGE) scan in a clinical protocol.

Methods: Fifty clinical patients underwent contrast-enhanced CS CMRA and conventional CMRA on a 3 T CMR scanner. After contrast injection, CS CMRA was scanned during the waiting time for LGE CMR. A conventional CMRA scan was performed after LGE CMR. We assessed acquisition times and coronary artery image quality for each segment on a 4-point scale. Visible vessel length, sharpness and diameter of right (RCA), left anterior descending (LAD), and left circumflex (LCX) coronary arteries were also quantitatively compared among the scans.

Results: All CS CMRA scans were successfully performed within the LGE waiting time. The median total scan time was 207 s $(163,259$ s) for CS and 785 s (698, 975 s) for conventional CMRA $(p<0.001)$. No significant differences were observed in image quality scores, vessel length measurements, sharpness, and diameter between CS and conventional CMRA.

Conclusions: We could achieve all CS CMRA scans within the LGE waiting time. Contrast-enhanced CS CMRA could considerably shorten the scan time while maintaining image quality compared with conventional CMRA.
\end{abstract}

Keywords: Coronary magnetic resonance angiography, Compressed sensing, Cardiovascular magnetic resonance

\section{Background}

Cardiovascular magnetic resonance (CMR) is capable of comprehensive assessment of the heart including anatomy and function, myocardial tissue characteristics, and coronary artery without radiation exposure [1-4]. Coronary magnetic resonance angiography (CMRA) has been developed over the past three decades as a possible noninvasive alternative for visualizing coronary arteries [5]. The balanced steady-state-free-precession (bSSFP) sequence has achieved great success in CMRA imaging

\footnotetext{
* Correspondence: tomozo0421@gmail.com

'Department of Radiology, Ehime University Graduate School of Medicine,

Shitsukawa, Toon, Ehime 791-0295, Japan

Full list of author information is available at the end of the article
}

at $1.5 \mathrm{~T}$ because of its intrinsic high blood signal intensity and blood-myocardial contrast [6]. Contrastenhanced whole-heart CMRA with segmented fast lowangle shot (FLASH) 3D spoiled gradient-echo sequence at $3 \mathrm{~T}$ also demonstrated better depiction of coronary segments compared with bSSFP coronary CMRA at 1.5 $\mathrm{T}$ [7]. The shortening of CMRA acquisition time has been made possible by novel multi-channel cardiac coils and high parallel imaging factors [8]. Nevertheless, a CMRA scan still requires a comparatively long acquisition time, and is affected by the issues of operator dependency and limited ease-of-use, which limit its general applicability in clinical practice.

(c) The Author(s). 2020 Open Access This article is distributed under the terms of the Creative Commons Attribution 4.0 International License (http://creativecommons.org/licenses/by/4.0/), which permits unrestricted use, distribution, and 
The recent development of the mathematical theory of compressed sensing (CS) has also been applied to CMR [9]. CS is based on reconstructing an image from an incompletely filled k-space $[10,11]$. The CS technique is known to reduce scan time considerably, and was recently applied to cine CMR [12-15]. Nakamura et al. reported that non-contrast CS CMRA could significantly shorten acquisition time, compared with conventional CMRA, in healthy subjects. In that study, almost all the CMRA scans were performed within 5 min [16].

In the clinical comprehensive CMR protocol, including cine CMR, perfusion CMR, late gadolinium enhancement (LGE) CMR, and CMRA, there is a waiting time of about $10 \mathrm{~min}$ after the injection of contrast medium for LGE CMR, and the CMRA scan is usually performed at the end of the protocol. We hypothesized that CMRA using CS could be routinely obtained within the LGE waiting time, thereby decreasing the total examination time and reducing patient burden. Therefore, the aim of this study was to verify the feasibility of CS CMRA scanning during the LGE waiting time in a clinical protocol.

\section{Materials and methods}

\section{Study population}

This is a prospective, two-center study. The study group consisted of 115 consecutive patients who were referred between August 2018 and March 2019 to undergo CMR for suspected cardiac disease. Exclusion criteria included non-contrast study, arrhythmia, patients with coronary stents or bypass grafts, and contraindications to CMR (claustrophobia, pacemaker). Sixty-five patients were excluded for these reasons, and 50 patients underwent CMRA (Fig. 1). The characteristics of the 50 patients are shown in Table 1. The institutional review board approved the study, and all participants gave written informed consent.

\section{CMR protocol}

CMR images were acquired using a $3 \mathrm{~T}$ whole-body CMR system (MAGNETOM Skyra; Siemens Healthineers, Erlangen, Germany). The CMR protocol typically included CS cine function, LGE, and CMRA (Fig. 2). Following the acquisition of CS cine imaging, gadobutrol $(0.1 \mathrm{mmol} / \mathrm{kg})$ was injected intravenously at a rate of 0.5 $\mathrm{mL} / \mathrm{s}$ with saline flash. LGE CMR was performed $10 \mathrm{~min}$ after the injection of contrast medium. CS CMRA, including preparatory steps such as detection of the data acquisition window and administration of nitroglycerin, was performed during the waiting time between contrast injection and LGE scan [17, 18], and conventional CMRA was performed after LGE CMR. If myocardial perfusion study was needed, gadobutrol $(0.05 \mathrm{mmol} / \mathrm{kg})$ was injected intravenously at a rate of $4 \mathrm{~mL} / \mathrm{s}$ with saline flash for each stress and rest perfusion imaging (total

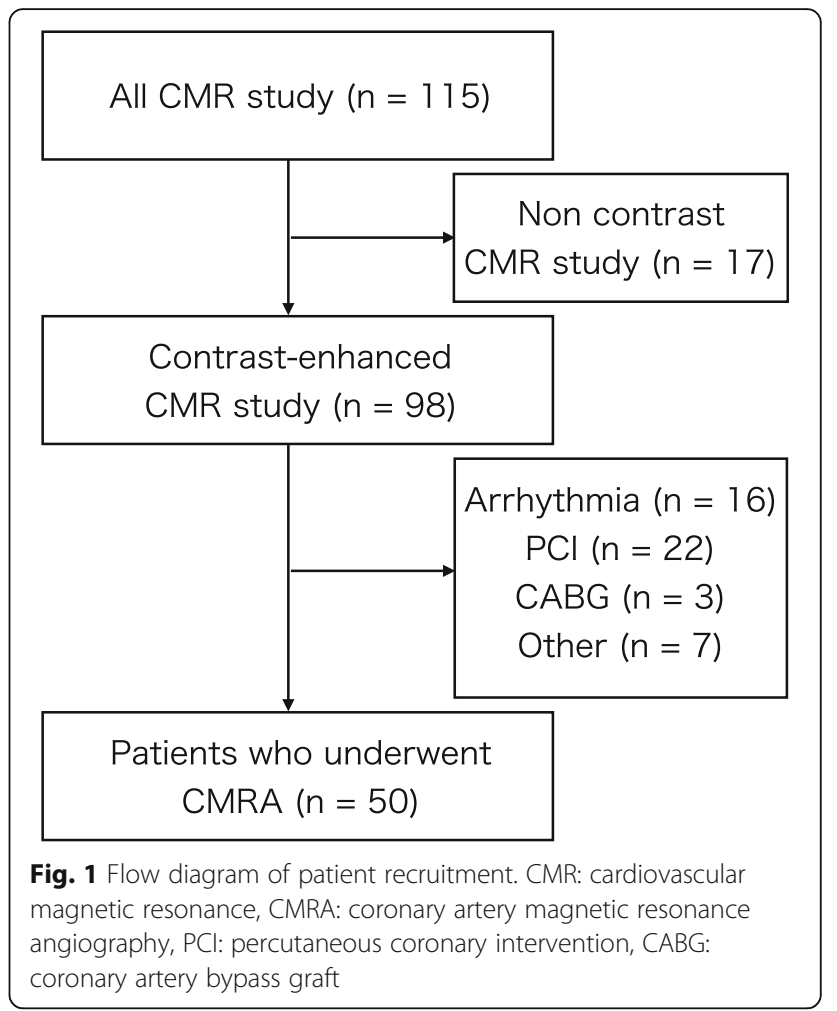

$0.1 \mathrm{mmol} / \mathrm{kg}$ ). LGE CMR was performed $10 \mathrm{~min}$ after the last contrast injection for rest perfusion imaging, and CS and conventional CMRA scans were performed as described above. If CS CMRA, including the preparatory steps, could not be completed within $10 \mathrm{~min}$ after contrast injection, the CS CMRA scan was terminated and considered failed, and the LGE scan was immediately performed.

Table 1 Characteristics of study population

\begin{tabular}{ll}
\hline Number & 50 \\
\hline Age $(\mathrm{y})$ & $60.5 \pm 16.0$ \\
Sex (female/male) & $21 / 29$ \\
Height $(\mathrm{cm})$ & $162.0 \pm 9.0$ \\
Weight $(\mathrm{kg})$ & $59.1 \pm 12.1$ \\
BMl $\left(\mathrm{kg} / \mathrm{m}^{2}\right)$ & $22.4 \pm 3.9$ \\
Hypertension & $20(40 \%)$ \\
Dyslipidemia & $14(28 \%)$ \\
Diabetes mellitus & $13(26 \%)$ \\
Smoking & $24(48 \%)$ \\
Family history of CAD & $17(34 \%)$ \\
Ischemia/Non ischemia & $14 / 36$ \\
\hline
\end{tabular}

The data are presented as the mean \pm standard deviation or as the number (\%) of subjects

$B M I$ Body mass index; $C A D$ coronary artery disease 


\section{Non-perfusion study}

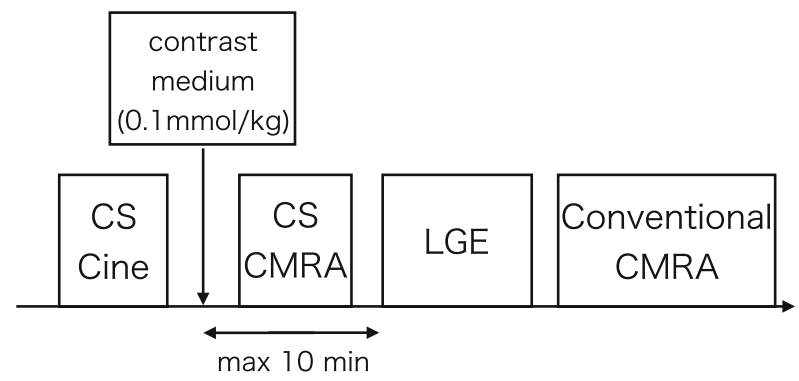

\section{Perfusion study}

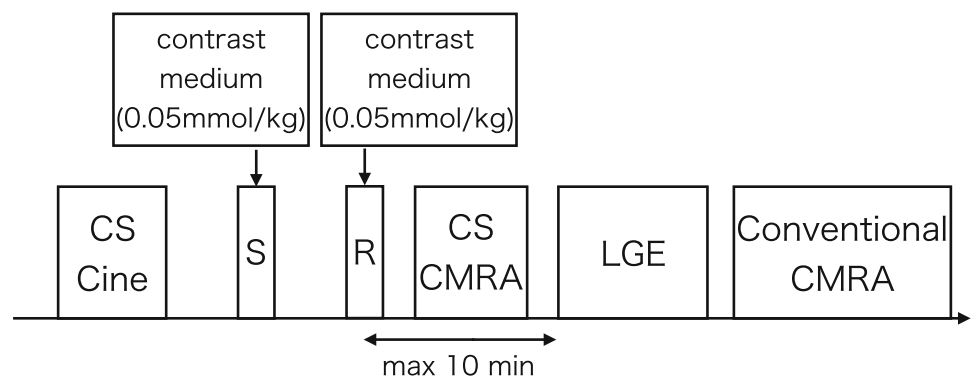

Fig. 2 Study protocol. CS: compressed sensing, CMRA: coronary magnetic resonance angiography, LGE: late gadolinium enhancement, S: Stress perfusion, R: Rest perfusion

\section{CMRA}

CMRA was obtained using electrocardiography (ECG)triggered and navigator-gated techniques. CMRA scans were obtained using a T2-prepared segmented 3D spoiled gradient echo sequence [19]. Conventional CMRA was compared to a CS prototype sequence. Imaging parameters are shown in Table 2. A nitroglycerin sublingual spray $(0.3 \mathrm{mg})$ was administered before each CMRA scan. A patient-specific data acquisition window length was determined in a 4-chamber cine sequence during either systole or diastole, according to the minimal motion of the right coronary artery (RCA) [20]. Respiratory motion was detected by placing a cross-pair navigator on the dome of the right hemi-diaphragm and data was only acquired in the expiratory phase. Respiratory acceptance rate was defined as the ratio of the acquired data within the setting window.

Data acquisition and image reconstruction CS CMRA Sparse incoherent sampling of the Cartesian phaseencoding plane was performed with a variable-density spiral phyllotaxis pattern for data acquisition [21]. The

Table 2 Image parameters

\begin{tabular}{|c|c|c|}
\hline & CS CMRA & Conventional CMRA \\
\hline Sequence type & spoiled gradient echo & spoiled gradient echo \\
\hline TR/TE (ms) & $3.2 / 1.4$ & $3.2 / 1.4$ \\
\hline $\mathrm{FOV}(\mathrm{mm})$ & $320 \times 258$ & $320 \times 258$ \\
\hline Matrix & $272 \times 220$ & $272 \times 220$ \\
\hline Actual voxel size (mm) & $1.2 \times 1.2 \times 1.8$ & $1.2 \times 1.2 \times 1.8$ \\
\hline Reconstruction voxel size (mm) & $1.2 \times 1.2 \times 0.9$ & $0.6 \times 0.6 \times 0.9$ \\
\hline $\begin{array}{l}\text { Acquisition window and Number of profiles acquired per } \\
\text { heartbeat }\end{array}$ & $\begin{array}{l}\text { adapted to the individual heart rate of the } \\
\text { subject }\end{array}$ & $\begin{array}{l}\text { same as possible for CS } \\
\text { imaging }\end{array}$ \\
\hline Bandwidth (Hz/pixel) & 593 & 613 \\
\hline Acceleration factor & 7.6 & 2 (GRAPPA) \\
\hline Acceptance window & $\pm 3.0 \mathrm{~mm}$ & $\pm 3.0 \mathrm{~mm}$ \\
\hline
\end{tabular}

CS compressed sensing; FOV field of view; GRAPPA generalized autocalibrating partially parallel acquisitions; TE echo time; TR repetition time 
acceleration factor was set to 7.6 compared with the fully sampled k-space. After data acquisition, images were reconstructed using CS reconstruction with a 3D spatial regularization using redundant Haar wavelets as described in [22]:

$$
\min _{\mathbf{x}}\|\mathbf{A x}-\mathbf{y}\|_{2}^{2}+\lambda\|\phi(x)\|_{1}
$$

where the first part of the equation optimizes the data fidelity of the estimated volume $\mathbf{x}$ with the acquired data y. A represents the system matrix containing the Fourier transform, coil sensitivity maps, and sampling pattern, and $\mathbf{x}$ the estimated image. The second term enforces a sparse representation of the image using a 11-norm regularization after the wavelet transformation $\phi()$. This cost function was optimized with a modified fast iterative shrinkage thresholding algorithm (FISTA) [23]. The second term was weighted with the regularization parameter $\lambda$, which was set to 0.0035 for both the phaseencoding and the slice-encoding direction regularization. For all datasets, the iterative reconstruction was terminated after 20 iterations.

\section{Qualitative image quality analysis}

Two radiologists with 10 years (reader 1 ) and 4 years (reader 2) of CMR experience independently graded image quality based on a four-point scale with respect to the border definition of coronary arteries, as follows: 1) severely blurred; 2) markedly blurred; 3) mildly blurred; 4) sharply defined. The artifact score was also graded with respect to wrap around, respiratory ghost, and cardiac ghost as follows: 1) severe artifacts; 2) some artifacts; 3) few artifacts; 4) no artifacts. Coronary segments were defined according to the classification of the American Heart Association [24], as follows: \#1: RCA proximal; \#2: RCA middle; \#: RCA distal; \#5: left anterior descending (LAD) main; \#6: LAD proximal; \#7: LAD middle; \#11: left circumflex (LCX) proximal; and \#13: LCX distal. Image quality and artifacts were assessed in the axial orientation. Image quality was evaluated for each segment and artifacts for the entire image. We used the image quality assessed by reader 1 and computed the inter-observer agreement with reader 2 for subsequent analyses.

\section{Quantitative vessel analysis}

CS and conventional CMRA images were transferred to an external workstation (SYNAPSE VINCENT, Fujifilm Corp., Ltd., Tokyo, Japan). A third radiologist, with 8 years of CMR experience and blinded to the origin of the images, performed curved planar reconstruction (CPR) of RCA, LAD, and LCX from each set of axial CMRA images, and measured the length of the vessels on CPR images semi-automatically using the workstation.
The sharpness and diameter of the vessels were evaluated on RCA\#1, LAD\#6, and LCX\#11. Signal intensity profiles along a user-defined line perpendicular to the major axes of the vessel were obtained. Vessel diameter was calculated as the full width at half between maximum and background [25]. To evaluate the sharpness of the vessel, the 20 and $80 \%$ points between the maximal and background signal intensities were first calculated for each side of the signal intensity profile. The distance in millimeters between the two points was then determined for each side. Vessel sharpness was defined as the inverse of the averaged distance of the two sides [26].

\section{Statistical analysis}

Continuous variables are presented as mean \pm standard deviation (SD) or as median (first quartile, third quartile). Acceptance rates, heart rates, sharpness, and diameter were compared between CS and conventional CMRA using paired t-tests. Scan times, image quality scores, artifact scores, and visible vessel length were compared between the two methods using the Wilcoxon matched-pairs signed-rank test. Correlation and agreement between the visible vessel lengths were assessed using linear regression and Bland-Altman analysis. The quadratic-weighted kappa test was used to evaluate the inter-observer agreement of image quality. A $P$-value $(p)$ $<0.05$ was considered as statistically significant, and Bonferroni correction was used to reduce the chance of obtaining false-positive results (type I errors) when multiple pair wise tests were performed in the quantitative vessel assessments (e.g., visible vessel length, sharpness, and diameter). Statistical analysis was carried out using the statistical software (JMP version 13; SAS Institute, Cary, North Carolina, USA).

\section{Results}

All CS and conventional CMRA scans were successfully performed for all 50 patients. The LCX proximal and distal segments (\#11, \#13) of one patient showed hypoplasia and were excluded from the analysis. In total, 149 vessels and 348 coronary artery segments from 50 patients were evaluated. The median total scan time, including navigator efficiency, was $207 \mathrm{~s}(163,259 \mathrm{~s})$ for CS and $785 \mathrm{~s}(698,975 \mathrm{~s})$ for conventional CMRA $(p<$ $0.001)$. The respiratory acceptance rate was $49.7 \pm 9.5 \%$ for CS CMRA, and $51.4 \pm 8.7 \%$ for conventional CMRA $(p=0.077)$. The heart rate was $66.5 \pm 11.4 \mathrm{bpm}$ for CS CMRA and $67.2 \pm 12.8 \mathrm{bpm}$ for conventional CMRA $(p=0.79)$. CS reconstruction was performed inline at the scanner at the end of the acquisition using graphical processing units (GPUs) on the MR reconstruction system. We could reconstruct the CS CMRA images in approximately $2 \mathrm{~min}$. 


\section{Qualitative image quality analysis}

Table 3 summarizes the image quality scores in each coronary artery segment. No significant differences were found in image quality scores and artifact scores between CS and conventional CMRA. The two radiologists showed good agreement in subjective image quality scores (Kappa $=0.72$ for CS CMRA, Kappa $=0.74$ for conventional CMRA) and artifact scores (Kappa $=0.79$ for CS CMRA, Kappa $=0.77$ for conventional CMRA). Figures 3 and 4 shows examples of CS and conventional CMRA for image analysis.

\section{Quantitative vessel analysis}

Table 4 summarizes the quantitative assessment of each coronary artery. No significant differences were observed in vessel length, sharpness, and diameter between CS and conventional CMRA. Representative CPR images of the two methods for the evaluation of visible vessel length are shown in Fig. 5. There was good agreement between CS and conventional CMRA for all measurements (Fig. 6).

\section{Discussion}

From this prospective, two-center study, we make the first report on the feasibility of contrast-enhanced CS CMRA in a clinical protocol. CS CMRA enabled a substantial reduction in acquisition time while maintaining image quality compared with conventional CMRA. Due to the short acquisition time, all CS CMRA scans were successfully performed within the waiting time $(10 \mathrm{~min})$ for LGE in the clinical protocol.

CMRA has emerged as a method capable of providing visualization of coronary arteries without ionizing radiation [27, 28]. Shorter acquisition times have become possible using bSSFP sequences, parallel imaging with multi-channel coils, respiratory gating with navigator echoes, and improved strategies for $\mathrm{k}$-space sampling $[29,30]$. Today, the major problems with CMRA are the

Table $\mathbf{3}$ Image quality scores

\begin{tabular}{llll}
\hline & CS CMRA & Conventional CMRA & $p$-value \\
\hline RCA proximal & $3.6 \pm 0.7$ & $3.5 \pm 0.7$ & 0.110 \\
RCA mid & $3.5 \pm 0.8$ & $3.6 \pm 0.7$ & 0.411 \\
RCA distal & $3.5 \pm 0.7$ & $3.5 \pm 0.6$ & 0.622 \\
LAD proximal & $3.7 \pm 0.5$ & $3.6 \pm 0.5$ & 0.376 \\
LAD mid & $3.6 \pm 0.8$ & $3.5 \pm 0.7$ & 0.316 \\
LAD distal & $3.3 \pm 0.8$ & $3.2 \pm 0.8$ & 0.600 \\
LCX proximal & $3.5 \pm 0.6$ & $3.5 \pm 0.6$ & 0.444 \\
LCX distal & $3.2 \pm 0.9$ & $3.3 \pm 0.7$ & 0.145 \\
Artifact & $3.8 \pm 0.4$ & $3.8 \pm 0.4$ & 0.730 \\
\hline
\end{tabular}

The data are presented as the mean \pm standard deviation $C S$ compressed sensing; $L A D$ left anterior descending artery; $L C X$ left circumflex artery; mid middle; $R C A$ right coronary artery relatively long acquisition time and image artifacts caused by motion instability during the scan. Recently, new rapid imaging methods such as self-navigation and CS have emerged which can overcome these problems $[18,31-33]$.

In our study, CS CMRA considerably shortened the acquisition time compared with conventional CMRA in clinical patients. In the clinical protocol, LGE CMR is commonly obtained $10 \mathrm{~min}$ after injection of contrast medium [34]. Our results showed that the examination time of CS CMRA, including preparatory steps such as the detection of the data acquisition window and the administration of nitroglycerin, is short enough to allow the scan to be performed within the waiting time between contrast injection and LGE scan.

The acquisition time of CS CMRA was very short and the acquisition data was smaller than conventional CMRA; therefore, the image quality of CS CMRA might be expected to be poorer. While a previous $3 \mathrm{~T}$ noncontrast study [16] showed inferior results from CS CMRA in terms of image quality and visible vessel length compared with conventional CMRA, our study did not reveal significant differences in either aspect. Furthermore, we found no significant difference in the quantitative evaluation of image quality as defined by sharpness. Several reasons were considered as to why the qualitative and quantitative evaluations with CS and conventional CMRA were not significantly different. Since conventional CMRA is performed at the end of the examination, it may be influenced by the patient's movement due to fatigue from the long CMR examination. In our study, CS CMRA could be performed at an early stage, thus decreasing patient fatigue and movement. The motion of the heart through the cardiac and respiratory cycles can also cause blurring and artifacts in CMR studies [35]. The short acquisition time of CS CMRA may also reduce the artifacts produced by unstable respiratory conditions and motion during the scanning (Fig. 4). Respiratory self-navigation has been proposed as an alternative approach to compensate for respiratory motion while also increasing scan efficiency $[18,31-33]$. Further shortening of the imaging time can be expected by the combination of CS and selfnavigation.

Differences in contrast agent concentration in the coronary artery at the time of imaging should also be considered. Contrast medium improves the signal-to-noise and contrast-to-noise ratios in CMRA, with an emphasis on the role of T1-shortening [36]. In our protocol, CS CMRA scans were performed immediately after injection of the contrast medium when the concentration of contrast agent was high. In comparison, since conventional CMRA was performed after LGE, the contrast agent had washed out over time and its concentration was relatively lower. 


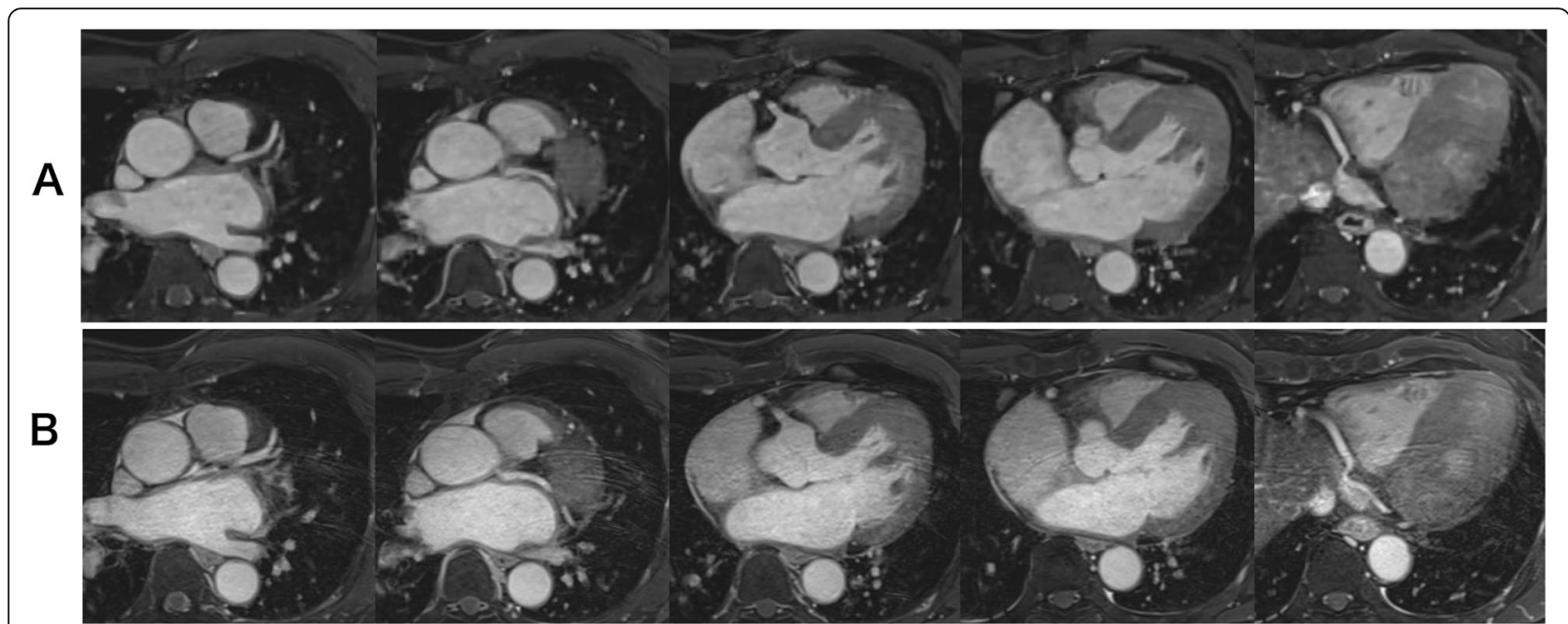

Fig. 3 Axial images of CS (a) and conventional (b) CMRA. Both image sets were acquired from a 63-year-old patient. Both observers rated the image quality as excellent (4 points) for both techniques. CS largely shortened the acquisition time ( 2 min $6 \mathrm{~s}$ for CS, 10 min $0 \mathrm{~s}$ for conventional)

Double-dose contrast medium $(0.2 \mathrm{mmol} / \mathrm{kg})$ is widely used for LGE [37]. However, in our study, single-dose (0.1 $\mathrm{mmol} / \mathrm{kg}$ ) medium was used because double-dose is not permitted in Japan. The single-dose was likely to reduce its concentration over time faster than the double-dose, leading to an advantage for CS CMRA that could be performed immediately after the injection of the contrast medium. In the CS technique, the contrast in the image plays a major part in the ability to reconstruct vastly undersampled images. High contrast often results in large distinct sparse coefficients [9]. These reasons may help to explain the lack of significant differences between CS and conventional CMRA for qualitative and quantitative evaluations. Contrast-enhanced CS CMRA could shorten the acquisition time considerably while maintaining image quality compared with conventional CMRA.
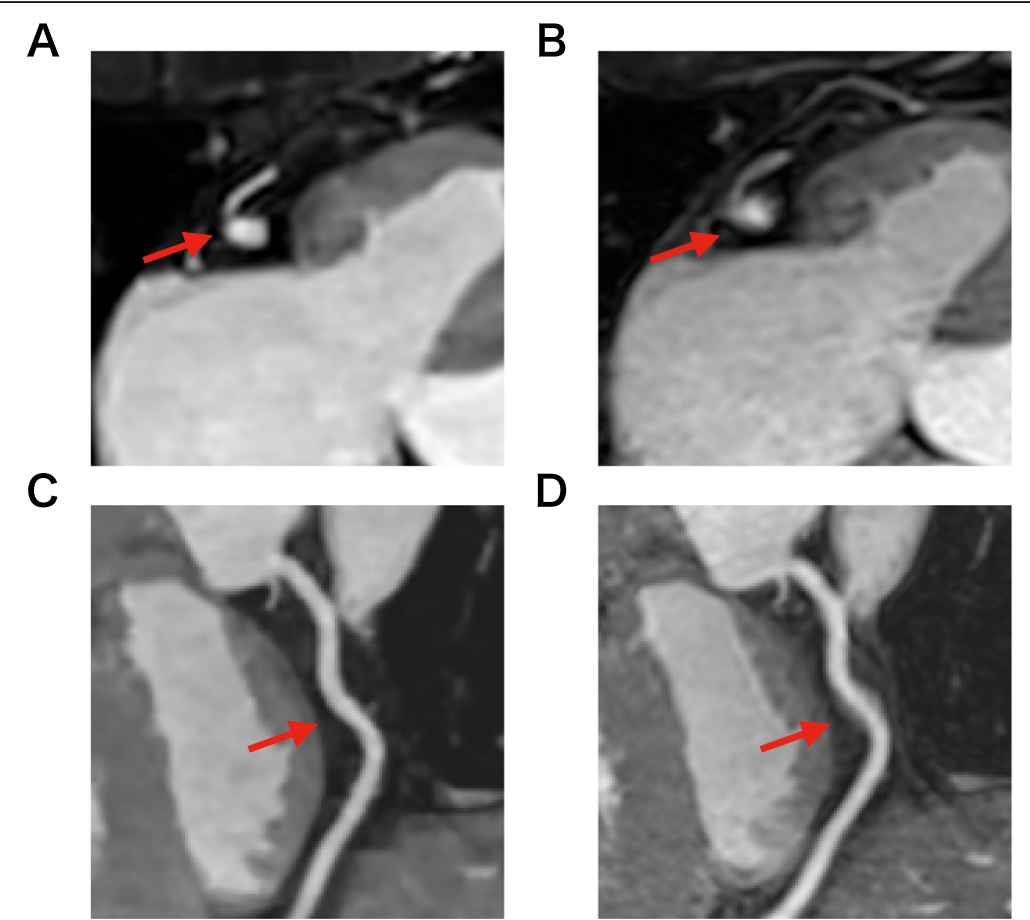

Fig. 4 Axial images and curved planar reconstruction for the right coronary artery (RCA) in the two techniques. Both image sets were acquired from a 65 -year-old patient. Conventional CMRA had motion artifacts on RCA (b, d), while CS CMRA did not (a, $\mathbf{c})$. Both observers rated the image quality for RCA mid as good (3 points) in conventional imaging (b) and excellent (4 points) in CS imaging (a) 
Table 4 Quantitative vessel assessment

\begin{tabular}{|c|c|c|c|}
\hline & CS CMRA & Conventional CMRA & $p$-value \\
\hline \multicolumn{4}{|c|}{ Vessel length (mm) } \\
\hline RCA & $149(128,161)$ & $146(132,163)$ & 0.189 \\
\hline LAD & $134(103,155)$ & $138(110,155)$ & 0.079 \\
\hline LCX & $91(80,111)$ & $93(82,114)$ & 0.153 \\
\hline \multicolumn{4}{|c|}{ Vessel sharpness $(1 / \mathrm{mm})$} \\
\hline RCA & $0.87 \pm 0.14$ & $0.85 \pm 0.16$ & 0.152 \\
\hline LAD & $0.90 \pm 0.15$ & $0.87 \pm 0.14$ & 0.090 \\
\hline LCX & $0.95 \pm 0.19$ & $0.94 \pm 0.16$ & 0.301 \\
\hline \multicolumn{4}{|c|}{ Vessel diameter (mm) } \\
\hline RCA & $4.0 \pm 0.6$ & $4.1 \pm 0.6$ & 0.514 \\
\hline LAD & $3.6 \pm 0.6$ & $3.5 \pm 0.6$ & 0.492 \\
\hline LCX & $3.2 \pm 0.5$ & $3.2 \pm 0.5$ & 0.701 \\
\hline
\end{tabular}

The data are presented as the mean \pm standard deviation or as the median (first quartile, third quartile)

$C S$ compressed sensing; $L A D$ left anterior descending artery; $L C X$ left circumflex artery; $R C A$ right coronary artery

In this study, we verified that adequate CS CMRA could be performed during the waiting time in a normal clinical protocol. Preparatory steps such as the detection of the data acquisition window and the administration of nitroglycerin were performed after contrast medium administration so as not to disturb the clinical examination. If preparatory steps are performed before contrast medium injection, it can maximize the blood gadolinium concentration during CS CMRA acquisition and allow more time for CS CMRA scanning.

In addition, CS can also potentially improve spatial resolution [38]. In our results, CS CMRA scan times were significantly shorter than the 10-min limit imposed by the LGE waiting time. Therefore, by spending more time on the CS CMRA acquisition, image quality can be improved, or isotropic resolution can be achieved.

In the future, further high resolution CMRA imaging may allow for detailed coronary artery assessment. We can expect such new protocols to replace conventional CMRA with CS CMRA performed during the waiting time. This protocol will significantly shorten the total CMR examination time and lead to a reduction in patient burden.

\section{Limitations}

Our study has several limitations. First, we evaluated only 50 patients and did not perform stenotic vessel evaluations. A previous study of contrast-enhanced conventional CMRA with a $3 \mathrm{~T}$ scanner showed detection of significant coronary artery disease (CAD) with good diagnostic performance [8]. We did not evaluate stenosis because coronary angiography was performed in only a few cases. However, in the quantitative evaluation of the diameter, there was no significant difference between CS and conventional CMRA, suggesting that CS had little effect on the diameter. Furthermore, we could present a representative case with significant CAD detected in CS CMRA as well as in conventional CMRA (Fig. 7). We expect that further studies will reveal the accuracy of CS CMRA in assessing significant CAD.

Second, complete blinding in the evaluation of image quality was not achieved. When we visually evaluated the images, we could readily distinguish CS coronary images from conventional coronary images, because CS

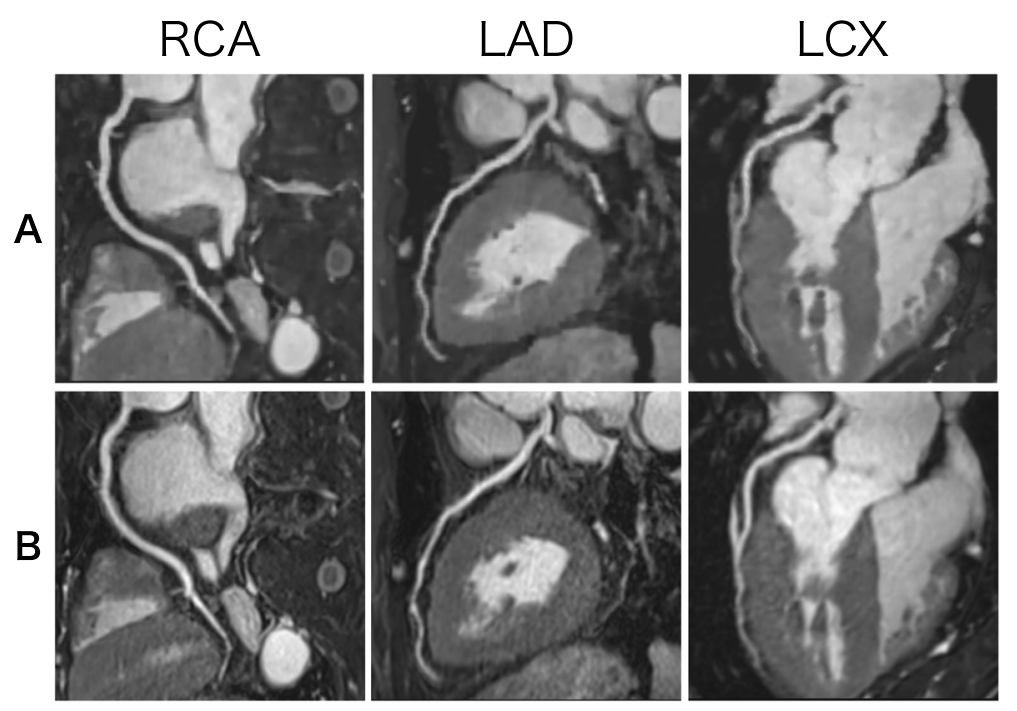

Fig. 5 Curved planar reconstruction of CS (a) and conventional (b) CMRA. Both image sets were acquired from a 63-year-old patient. Visible vessel length was similar in the two techniques (RCA: CS $154 \mathrm{~mm}$ vs. conventional $156 \mathrm{~mm}$; left anterior descending (LAD): CS $148 \mathrm{~mm}$ vs. conventional $149 \mathrm{~mm}$; left circumflex (LCX): CS $127 \mathrm{~mm}$ vs. conventional $129 \mathrm{~mm}$ ) 

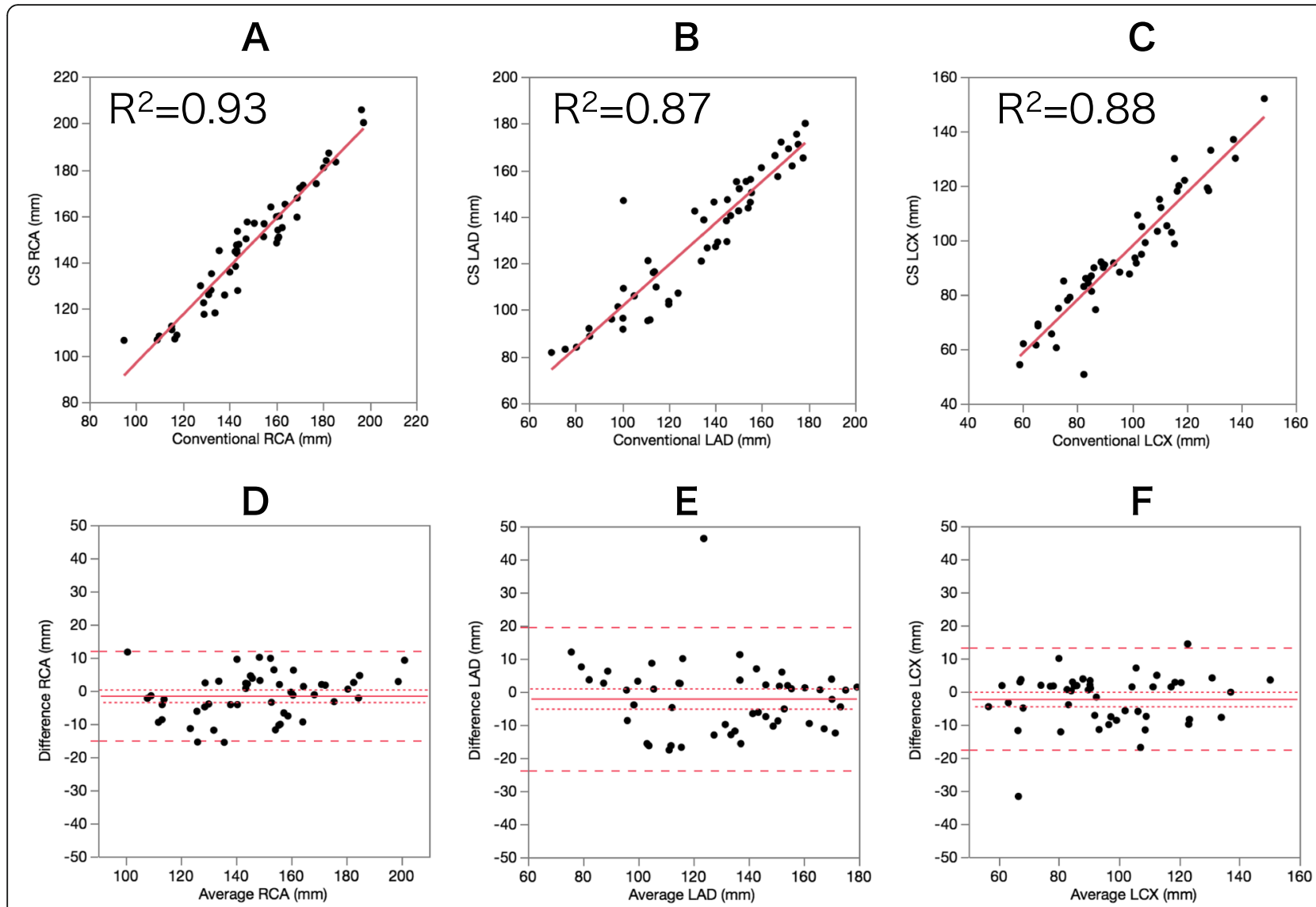

Fig. 6 Scatter plots and Bland-Altman plots for the visible vessel length in the two techniques. In Bland-Altman plots, the solid line indicates the mean difference between the two techniques; the long-dashed lines indicate the corresponding double standard deviation intervals; and the short-dashed lines indicate the $95 \%$ confidence interval of the mean difference. a/d: right coronary artery, $\mathbf{b} / \mathbf{e}$ : left anterior descending artery, $\mathbf{c} / \mathbf{f}$ : left circumflex artery

CMRA shows less noise and has a slightly pixelated and textured appearance compared with conventional CMRA. This factor may have contributed to bias in the evaluation of coronary image quality.

Third, in our protocol, all CS CMRA scans were performed first, followed by conventional CMRA scans; random sequence ordering was not performed. This may have been advantageous for CS CMRA due to the higher concentration of contrast agent and less patient movement from fatigue. As CS and conventional CMRA could not be compared under the same conditions, there may be a bias. However, if the conventional CMRA scan
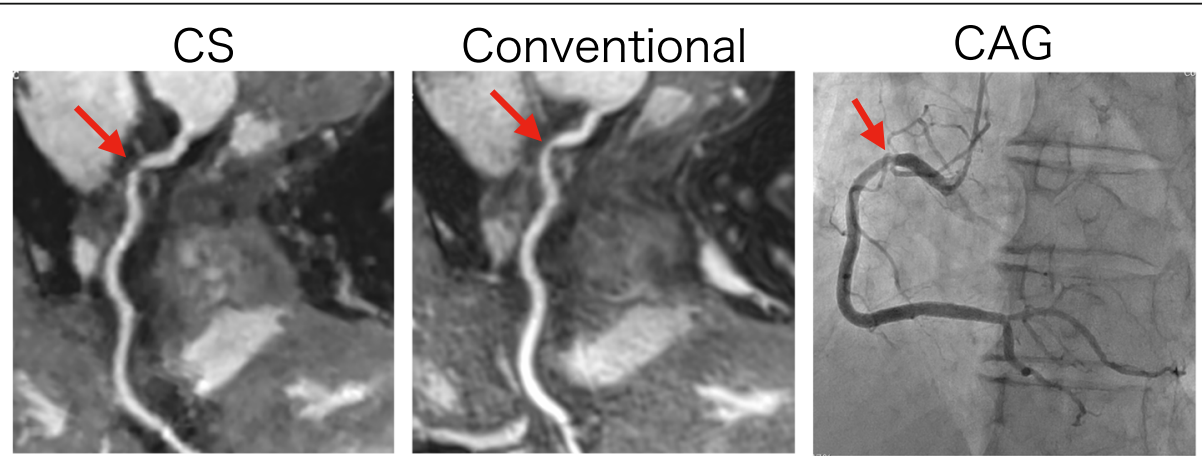

Fig. 7 A 59-year-old patient with chest pain. Significant coronary artery stenosis in the RCA is observed in the CS (a) and conventional (b) CMRA, with good correlation with coronary angiography (c) 
is performed first, the time to LGE scan after contrast medium ejection will be longer, which will decrease the contrast concentration and lead to a reduction in LGE image quality in clinical practice [39]. Since the acquisition time is short, CS CMRA examination can be performed in the waiting time without deteriorating LGE imaging.

Fourth, there were few cases of obesity within our Japanese patient cohort. When CMRA examination is performed with a Western population where body mass index is often much higher in patients suspected of CAD, the image quality may be decreased due to poor fat saturation and aliasing [40]. Larger fields of view also require longer acquisition time assuming the image resolution is unchanged. Therefore, our results may be not always applied to a Western patient population. In addition, the abdominal belt technique, which suppresses breathingrelated motion of the diaphragm, was not used in the current study so as to not disturb the clinical examination. The use of an abdominal belt is expected to further improve image quality and shorten acquisition time $[5,41]$.

\section{Conclusions}

The acquisition time of CS CMRA is short enough to allow the scan to be performed within the waiting time for LGE, thereby shortening the protocol time while maintaining image quality as compared with conventional CMRA performed after LGE imaging. These findings suggest the possibility of a faster CMR protocol.

\section{Supplementary information}

Supplementary information accompanies this paper at https://doi.org/10. 1186/s12968-020-0601-0.

Additional file 1. Histogram distribution of the image quality scores

\section{Abbreviations \\ bSSFP: Balanced steady state free precession; CAD: Coronary artery disease; CMR: Cardiovascular magnetic resonance; CMRA: Coronary magnetic resonance angiography; CPR: Curved planar reconstruction; CS: Compressed sensing; ECG: Electrocardiogram; FISTA: Fast iterative shrinkage threshold algorithm; FLASH: Fast low-angle shot; GPU: Graphical processing unit; LAD: Left anterior descending coronary artery; LCX: Left circumflex coronary artery; LGE: Late gadolinium enhancement; RCA: Right coronary artery}

\section{Acknowledgements}

The authors are grateful to Yoshiaki Komori of Siemens Healthcare K.K. (Tokyo, Japan) for the optimization of the sequence parameters and image quality in the study.

\section{Authors' contributions}

$\mathrm{KH}$ and TK conceived the study, analyzed qualitative image quality analysis, and drafted the manuscript. MN performed quantitative vessel analysis and contributed to the revision of the manuscript. YT and NK performed the statistical data analyses. TK, RO, AK, KW revised the manuscript and participated in the scientific discussion during the study. OY participated in the design of the study and coordination and helped to revise the manuscript. MS contributed to the sequence development, implementation on the scanner and helped to revise the manuscript. CF contributed to the sequence development and implementation on the scanner and helped to revise the manuscript. TM assisted in the interpretation of the results and helped to revise the manuscript. All authors read and approved the final manuscript.

\section{Funding}

Not applicable.

\section{Availability of data and materials}

Not applicable.

\section{Ethics approval and consent to participate}

The study protocol was approved by the Ethical Review Board in Saiseikai Matsuyama Hospital and Ehime University Graduate School of Medicine (1807008). All participants in this study gave written consent to participate and to publish.

\section{Consent for publication}

All participants in this study gave written consent to participate and to publish.

\section{Competing interests}

The authors declare that they have no competing interests.

\section{Author details}

${ }^{1}$ Department of Radiology, Ehime University Graduate School of Medicine, Shitsukawa, Toon, Ehime 791-0295, Japan. ²Department of Radiology, Saiseikai Matsuyama Hospital, 880-2, Yamanishi, Matsuyama, Ehime 791-8026, Japan. ${ }^{3}$ Department of Cardiology, Saiseikai Matsuyama Hospital, 880-2, Yamanishi, Matsuyama, Ehime 791-8026, Japan. ${ }^{4}$ Department of Cardiology, Ehime University Graduate School of Medicine, Shitsukawa, Toon, Ehime 791-0295, Japan. ${ }^{5}$ Siemens Healthcare GmbH, Allee am Roethelheimpark 2, 91052 Erlangen, Germany.

Received: 28 June 2019 Accepted: 9 January 2020

Published online: 13 February 2020

\section{References}

1. Karamitsos TD, Francis JM, Myerson S, Selvanayagam JB, Neubauer S. The role of cardiovascular magnetic resonance imaging in heart failure. J Am Coll Cardiol. 2009;54:1407-24.

2. Greenwood JP, Maredia N, Younger JF, Brown JM, Nixon J, Everett CC, et al. Cardiovascular magnetic resonance and single-photon emission computed tomography for diagnosis of coronary heart disease (CE-MARC): a prospective trial. Lancet. 2012;379:453-60.

3. Alfakih $\mathrm{K}$, Plein $\mathrm{S}$, Thiele $\mathrm{H}$, Jones T, Ridgway JP, Sivananthan MU. Normal human left and right ventricular dimensions for MRI as assessed by turbo gradient echo and steady-state free precession imaging sequences. J Magn Reson Imaging. 2003;17:323-9.

4. Kim WY, Danias PG, Stuber M, Flamm SD, Plein S, Nagel E, et al. Coronary magnetic resonance angiography for the detection of coronary stenoses. $\mathrm{N}$ Engl J Med. 2001;345:1863-9.

5. Kato S, Kitagawa K, Ishida N, Ishida M, Nagata M, Ichikawa Y, et al. Assessment of coronary artery disease using magnetic resonance coronary angiography: a national multicenter trial. J Am Coll Cardiol. 2010;56:983-91.

6. Finn JP, Nael K, Deshpande V, Ratib O, Laub G. Cardiac MR imaging: state of the technology. Radiology. 2006;241:338-54.

7. Liu X, Bi X, Huang J, Jerecic R, Carr J, Li D. Contrast-enhanced whole-heart coronary magnetic resonance angiography at $3.0 \mathrm{~T}$ : comparison with steady-state free precession technique at 1.5 T. Invest Radiol. 2008;43:663-8.

8. Yang Q, Li K, Liu X, Du X, Bi X, Huang F, et al. 3.0T whole-heart coronary magnetic resonance angiography performed with 32-channel cardiac coils: a single center experience. Circ Cardiovasc Imaging. 2012;5:573-9.

9. Lustig M, Donoho D, Pauly JM. Sparse MRI: the application of compressed sensing for rapid MR imaging. Magn Reson Med. 2007;58:1182-95.

10. Candès E, Romberg J, Tao T. Robust uncertainty principles: exact signal reconstruction from highly incomplete frequency information. IEEE Trans Inf Theory. 2006;52:489-509.

11. Donoho D. Compressed sensing. IEEE Trans Inf Theory. 2006;52:1289-306.

12. Kido T, Kido T, Nakamura M, Watanabe K, Schmidt M, et al. Compressed sensing real-time cine cardiovascular magnetic resonance: accurate 
assessment of left ventricular function in a single- breath-hold. J Cardiovasc Magn Reson. 2016;18:50

13. Vincenti G, Monney P, Chaptinel J, et al. Compressed sensing single-breathhold CMR for fast quantification of LV function, volumes, and mass. JACC Cardiovasc Imaging. 2014;7:882-92.

14. Wech T, Lemke A, Medway D, Stork LA, Lygate CA, Neubauer S, et al. Accelerating cine-MR imaging in mouse hearts using compressed sensing. J Magn Reson Imaging. 2011;34:1072-9.

15. Roy CW, Seed M, Kingdom JC, Macgowan CK. Motion compensated cine CMR of the fetal heart using radial undersampling and compressed sensing. J Cardiovasc Magn Reson. 2017;19:29.

16. Nakamura M, Kido T, Kido T, Watanabe K, Schmidt M, Forman C, et al. Noncontrast compressed sensing whole-heart coronary magnetic resonance angiography at 3T: a comparison with conventional imaging. Eur J Radiol. 2018;104:43-8

17. Börnert P, Koken P, Nehrke K, Eggers H, Ostendorf P. Water/fat-resolved whole-heart Dixon coronary MRA: an initial comparison. Magn Reson Med. 2014;71:156-63.

18. Piccini D, Monney P, Sierro C, Coppo S, Bonanno G, van Heeswijk RB, et al. Respiratory self-navigated postcontrast whole-heart coronary MR angiography: initial experience in patients. Radiology. 2014;270:378-86.

19. Nezafat R, Stuber M, Ouwerkerk R, Gharib AM, Desai MY, Pettigrew RI. B1insensitive T2 preparation for improved coronary magnetic resonance angiography at 3 T. Magn Reson Med. 2006;55:858-64.

20. Sakuma H, Ichikawa Y, Suzawa N, Hirano T, Makino K, Koyama N, et al. Assessment of coronary arteries with total study time of less than 30 minutes by using whole-heart coronary MR angiography. Radiology. 2005: 237:316-21.

21. Forman C, Piccini D, Grimm R, Hutter J, Hornegger J, Zenge MO. Highresolution 3D whole-heart coronary MRA: a study on the combination of data acquisition in multiple breath-holds and 1D residual respiratory motion compensation. MAGMA. 2014;27:435-43.

22. Liu J, Rapin J, Chang T, Lefebvre A, Zenge M, Mueller E, et al. Dynamic cardiac MRI reconstruction with weighted redundant Haar wavelets. ISMRM. 2012;20:178.

23. Beck A, Teboulle M. A fast iterative shrinkage-thresholding algorithm for linear inverse problems. SIAM J Imaging Sci. 2009;2:183-202.

24. Austen WG, Edwards JE, Frye RL, Gensini GG, Gott VL, Griffith LS, et al. A reporting system on patients evaluated for coronary artery disease. Report of the ad hoc Committee for Grading of coronary artery disease, council on cardiovascular surgery, American Heart Associate. Circulation. 1975;51:5-40.

25. Shea S, Kroeker R, Deshpande V, Laub G, Zheng J, Finn J, et al. Coronary artery imaging: $3 \mathrm{D}$ segmented $\mathrm{k}$-space data acquisition with multiple breath-holds and real-time slab following. J Magn Reson Imaging. 2001;13: $301-7$.

26. Li D, Carr JC, Shea SM, Zheng J, Deshpande VS, Wielopolski PA, et al. Coronary arteries: magnetization-prepared contrast-enhanced threedimensional volume-targeted breath-hold MR angiography. Radiology. 2001; 219:270-7.

27. Weber OM, Martin AJ, Higgins CB. Whole-heart steady-state free precession coronary artery magnetic resonance angiography. Magn Reson Med. 2003; 50:1223-8.

28. Stuber M, Botnar RM, Fischer SE, Lamerichs R, Smink J, Harvey P, et al. Preliminary report on in vivo coronary MRA at 3 tesla in humans. Magn Reson Med. 2002:48:425-9.

29. McCarthy RM, Shea SM, Deshpande VS, Green JD, Pereles FS, Carr JC, et al. Coronary MR angiography: true FISP imaging improved by prolonging breath holds with preoxygenation in healthy volunteers. Radiology. 2003; 227:283-8.

30. Ishida M, Kato S, Sakuma H. Cardiac MRI in ischemic heart disease. Circ J. 2009;73:1577-88

31. Bastiaansen JAM, van Heeswijk RB, Stuber M, Piccini D. Noncontrast freebreathing respiratory self-navigated coronary artery cardiovascular magnetic resonance angiography at $3 \mathrm{~T}$ using lipid insensitive binomial off- resonant excitation (LIBRE). J Cardiovasc Magn Reson. 2019;21:38.

32. Piccini D, Feng L, Bonanno G, Coppo S, Yerly J, Lim RP, et al. Fourdimensional respiratory motion-resolved whole heart coronary MR angiography. Magn Reson Med. 2017;77:1473-84.

33. Luo J, Addy NO, Ingle RR, Baron CA, Cheng JY, H BS, et al. Nonrigid motion correction with 3D image-based navigators for coronary MR angiography. Magn Reson Med. 2017;77:1884-93.
34. Kramer CM, Barkhausen J, Flamm SD, Kim RJ, Nagel E. Society for Cardiovascular Magnetic Resonance Board of trustees task force on standardized protocols. Standardized cardiovascular magnetic resonance (CMR) protocols 2013 update. J Cardiovasc Magn Reson. 2013;15:91.

35. Scott AD, Keegan J, Firmin DN. Motion in cardiovascular MR imaging. Radiology. 2009;250:331-51.

36. Lorenz CH, Johansson LO. Contrast-enhanced coronary MRA. J Magn Reson Imaging. 1999;10:703-8.

37. Kim RJ, Wu E, Rafael A, Chen EL, Parker MA, Simonetti O, et al. The use of contrast-enhanced magnetic resonance imaging to identify reversible myocardial dysfunction. N Engl J Med. 2000;343:1445-53.

38. Basha TA, Akcakaya M, Liew C, Tsao CW, Delling FN, Addae G, et al. Clinical performance of high-resolution late gadolinium enhancement imaging with compressed sensing. J Magn Reson Imaging. 2017;46:1829-38.

39. D'Angelo T, Grigoratos C, Mazziotti S, Bratis K, Pathan F, Blandino A, et al. High-throughput gadobutrol-enhanced CMR: a time and dose optimization study. J Cardiovasc Magn Reson. 2017;19:83.

40. Graves MJ, Mitchell DG. Body MRI artifacts in clinical practice: a physicist's and radiologist's perspective. J Magn Reson Imaging. 2013;38:269-87.

41. Ishida M, Schuster A, Takase S, Morton G, Chiribiri A, Bigalke B, et al. Impact of an abdominal belt on breathing patterns and scan efficiency in wholeheart coronary magnetic resonance angiography: comparison between the UK and Japan. J Cardiovasc Magn Reson. 2011;13:71.

\section{Publisher's Note}

Springer Nature remains neutral with regard to jurisdictional claims in published maps and institutional affiliations.
Ready to submit your research? Choose BMC and benefit from:

- fast, convenient online submission

- thorough peer review by experienced researchers in your field

- rapid publication on acceptance

- support for research data, including large and complex data types

- gold Open Access which fosters wider collaboration and increased citations

- maximum visibility for your research: over $100 \mathrm{M}$ website views per year

At BMC, research is always in progress.

Learn more biomedcentral.com/submissions 